\title{
The User Interface for the ATLAS Trigger and Its Use in Physics Studies
}

\author{
T. Eifert, N. Berger, T. Bold, S. George, R. Goncalo, J. Haller, J. Stelzer, and M. Wielers
}

\begin{abstract}
The ATLAS detector at the Large Hadron Collider (LHC) will be exposed to proton-proton collisions at a rate of $40 \mathrm{MHz}$. This rate needs to be reduced to an output rate of $100-200 \mathrm{~Hz}$, compatible with the foreseen storage and analysis capability. To achieve this while retaining the most interesting physics, the trigger uses novel techniques, such as seeded, step-wise reconstruction and early rejection. As the luminosity increases more potentially interesting events will be produced than can be kept for analysis. To maximise the physics reach within the available bandwidth, the trigger menu will need to adapt to the increasing luminosity according to the ATLAS physics program. To study the bias introduced by the trigger selection, detailed online information needs to be used in physics analyses. A user interface was developed that, for each recorded event, allows easy access to information produced by the trigger as well as to its configuration. A first version of this interface was made available earlier this year and is now routinely used in physics studies.

We describe the ATLAS trigger operation and present in particular the trigger-user interface, focusing on the accessibility it provides to both online quantities and the trigger configuration. We also discuss the impact of the trigger selection on ATLAS physics studies relevant for the initial phase of $\mathrm{LHC}$ running.
\end{abstract}

\section{INTRODUCTION}

$\mathbf{T}$ HE Large Hadron Collider (LHC) at CERN, Geneva, is nearing completion. It will ultimately provide protonproton collisions at a centre-of-mass energy of $14 \mathrm{TeV}$, a design luminosity of $10^{34} \mathrm{~cm}^{-2} \mathrm{~s}^{-1}$ and a bunch-crossing rate of $40 \mathrm{MHz}$. ATLAS is a general purpose experiment for the LHC which is described in [1], [2]. The primary physics goals of ATLAS are to understand the mechanism for electroweak symmetry breaking and to search for new physics at the $\mathrm{TeV}$ energy scale. The trigger and data acquisition (T/DAQ) system must work in the challenging environment of $\sim 10^{9} \mathrm{p}$ $\mathrm{p}$ interactions per second and read a large number $\left(\sim 10^{8}\right)$ of electronics channels of the ATLAS detector. This very large

Manuscript received November ??, 2007.

T. Eifert is with the Geneva University (DPNC) Nuclear and Corpuscular Physics Department, (e-mail: Till.Eifert@cern.ch).

N. Berger is with the Institut National de Physique Nucleaire et de Physique des Particules (IN2P3-LAPP) Laboratoire d'Annecy-le-Vieux de Physique des Particules, (e-mail: Nicolas.Berger@cern.ch).

T. Bold is with the University of California. Irvine Department of Physics and Astronomy, (e-mail: Tomasz.Bold@ cern.ch).

S. George is with Royal Holloway, University of London, Department of Physics (e-mail: S.George@rhul.ac.uk).

R. Goncalo is with Royal Holloway, University of London, Department of Physics (e-mail: R.Goncalo@rhul.ac.uk).

J. Haller is with the Hamburg University and DESY (e-mail: Johannes.Haller@physik.uni-hamburg.de).

J. Stelzer is with CERN, (e-mail: Joerg.Stelzer@cern.ch).

M. Wielers is with Rutherford Appleton Laboratory, Abbingdon, United Kingdom (email: mwielers@mail.cern.ch) volume of raw data produced by the experiment must be reduced to the $\sim 300 \mathrm{MB} / \mathrm{s}$ which can be sustained to mass storage, while efficiently retaining rare physics signatures for off-line analysis. To achieve this, ATLAS has designed a threelevel trigger system (see Fig. 1) [3].

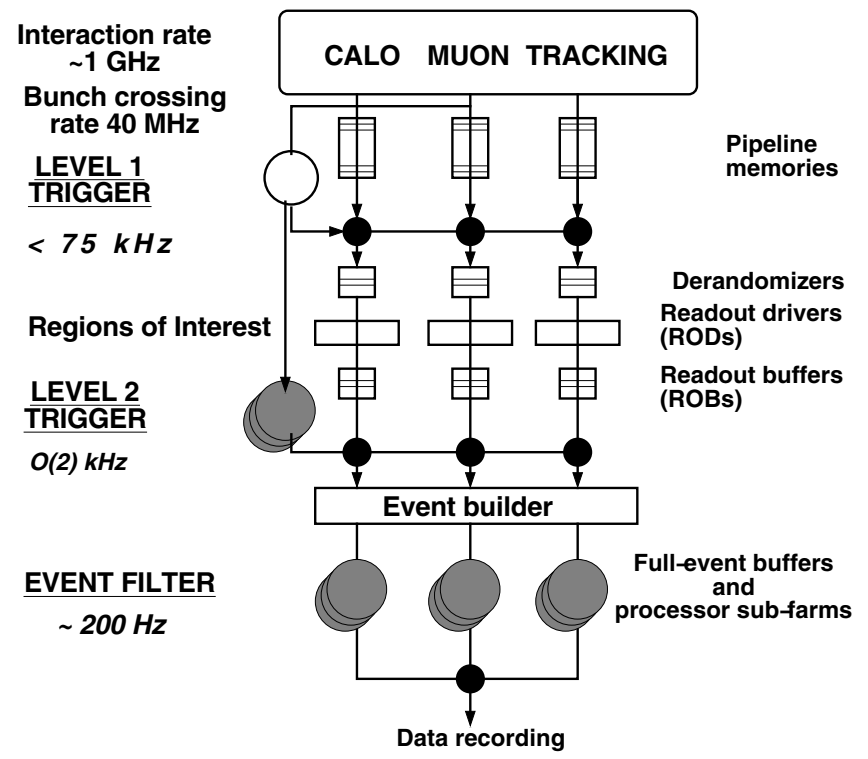

Fig. 1. The ATLAS T/DAQ system. The HLT runs in the LVL2 and EF processors which are shown as filled in circles.

The first level trigger (LVL1) is implemented in custom electronics (mainly ASICs and FPGAs). Its decision is based on relatively coarse data from two subsystems, the calorimeters and dedicated muon trigger stations. Event selection is based mainly on inclusive high- $p_{t}$ objects (muons, electromagnetic/tau/hadronic clusters, jet clusters, missing and scalar transverse energy sums) whose trigger thresholds are programmable. During the LVL1 latency of $2.5 \mu \mathrm{s}$ the data of all sub-detectors are kept in pipeline memories. For accepted events, the geometrical location of the objects, Regions of Interest (RoIs), and the thresholds they passed, are sent to the second level trigger (LVL2) and the data are then transferred from the pipeline memories to the Read-Out Buffers (ROBs). LVL1 reduces the event rate from the initial $40 \mathrm{MHz}$ to about $75 \mathrm{kHz}$.

The High Level Trigger (HLT) is a software-based trigger, running on farms built from commodity computing and network technology. It is an asynchronous, distributed system. The HLT is subdivided into LVL2 and the Event Filter (EF). LVL2 reduces the output rate to around $2 \mathrm{kHz}$. The EF should further reduce the rate to $\sim 200 \mathrm{~Hz}$. Both levels have access 
to the full granularity of all the detector data and follow the principle of further refining the signatures identified at LVL1. LVL2 must retrieve events fragments from the ROBs via Ethernet. To reduce the data transfer to a few percent of the full event size, it uses only data in RoIs identified by LVL1. LVL2 algorithms are highly optimised for speed. If LVL2 accepts an event, all the fragments from the ROBs are combined and sent to one EF processor for further consideration. The EF further refines the classification of LVL2, using the extra time to run more complex algorithms, often based on the same tool set as off-line reconstruction. It also benefits from more detailed calibration and alignment than LVL2.

\section{TRIGgER CONFIGURATION}

A detailed description of the ATLAS trigger configuration can be found elsewhere [4] but the essential concepts needed to understand the trigger-user interface are introduced here.

TABLE I

SAMPLE TRIGGER MENU TABLE. PS INDICATES "PRE-SCALE” AND PT MEANS "PASS-THROUGH".

$\begin{array}{llll}\text { Generic name } & \text { LVL1 item } & \text { LVL2 chain } & \text { EF chain } \\ \text { e5 } & \text { L1_EM3 (PS) } & \text { L2_e5 } & \text { EF_e5 } \\ \text { e5_PT } & \text { L1_EM3 (PS) } & \text { L2_e5_PT } & \text { EF_e5_PT } \\ \text { e10 } & \text { L1_EM8 } & \text { L2_e10 } & \text { EF_e10 } \\ \text { e10 } & \text { L1_EM8 } & \text { L2_g10 } & \text { EF_g10 } \\ \text { 2e10 } & \text { L1_2EM8 } & \text { L2_2e10 } & \text { EF_2e10 } \\ \text { e20_XE12 } & \text { L1_EM18_XE12 } & \text { L2_e20_Xe12 } & \text { EF_e20_xe12 } \\ \text { XE12 } & \text { L1_XE12 (PS) } & \text { L2_Xe12_PT } & \text { EF_xe12_PT }\end{array}$

Table I shows a small selection from a draft trigger menu designed for start-up of the LHC. The full menu contains electron (e), photon ( $g$ ), muon (mu), tau (tau), jet (j), b-jet (b), missing energy (xe), total energy (te), jet energy (je) and B-physics triggers, in single, multiple and combined triggers, with various thresholds each. It has low threshold, pre-scaled (PS) and pass-through (PT) items to help understand and cross-check the trigger. The numbers in the trigger names represent nominal thresholds in GeV. At LVL1, EM refers to electromagnetic clusters; electrons and photons cannot be separated at this level because there is no inner tracker data available.

Each trigger has a generic name, with a corresponding LVL1 item, LVL2 chain and EF chain. These HLT chains are central to the design of the the HLT. A chain is composed of several steps. These are the steps needed to confirm or reject this particular trigger in an event. Each step represents one or more algorithms. Breaking down the trigger chains into several steps realises the principle of early-rejection.

LVL1 has a hardware limit of 256 LVL1 items. Whereas the number of trigger chains in the two HLT levels is only limited by the assigned space inside the raw data for the chain identifier (chain counter). Often one LVL1 item seeds more than one LVL2 chain, as for instance the electromagnetic cluster item (L1_EM8) initiates both, the electron (L2_e10) and the photon trigger (L2_g10) chains in LVL2.

\section{DATA FlOW OF TRIgGER CONFIGURATION DATA}

The three trigger levels in ATLAS obtain all of their configuration information from a special trigger database
(TriggerDB). This TriggerDB provides all information that is needed to run the full online trigger. It includes the complete trigger menu with all pre-scaling and pass-through factors, all LVL1 hardware settings, and all HLT algorithm properties. Once a trigger configuration is put into the TriggerDB, it will be saved forever. This enables for example re-running of the trigger in offline data using the correct trigger setup of the time of data taking.

A subset of the trigger configuration is also saved into the ATLAS conditions database. In contrast to the TriggerDB, the conditions database is accessible from outside the ATLAS online computer nodes. The trigger configuration data in the conditions database is sufficient to fully interpret all trigger results in the raw data stream.

Monte Carlo production, however, requires the full TriggerDB to be shipped to the production site.

\section{TRIGGER RESUlt}

Trigger data can be divided into event-wise and run-wise information. The raw data stream contains event-wise trigger information, namely the encoded trigger decision. This trigger decision comprises:

- LVL1: acceptance flags for all 256 LVL1 items before and after the application of prescale and veto, thus filling $3 \times 256$ bits

- HLT: acceptance flags for each chain before and after the application of prescale and pass-through. HLT trigger chains are identified by a short integer (chain counter). The size depends on the number of used chains.

- Index of last successfully processed step of each used HLT chain.

- Error codes of each used HLT chain.

- Trigger data objects, for example electromagnetic clusters, muon tracks and so on.

- Dynamic link structure to link the produced trigger data objects to HLT algorithms.

Trigger data not changing on an event basis, i.e. configuration data, is kept apart from the event-wise data to save disk space. The following list shows the trigger configuration data that is needed to interpret the raw trigger data.

- Mapping of trigger names (strings) to bit positions or chain counters for LVL1 and HLT triggers respectively. This allows for access to the trigger decision using human readable trigger names.

- Full definition of the trigger chains, including all steps and algorithms. This is the static extension of the dynamic link structure. Combining the two allows to retrieve trigger data objects by chain name.

- LVL1 and HLT prescale, LVL1 veto, and HLT passthrough factors

\section{TRIGGER INFORMATION STORED IN ANALYSIS DATA OBJECT FILES}

In the ATLAS offline reconstruction, the raw data is processed resulting in either event summary data (ESD) or analysis data object (AOD) files. As a part of this ESD or AOD reconstruction, all raw trigger data is combined and stored in 
each event in a trigger decision class. The run-wise trigger configuration data from the conditions database is saved into the header of each file. Configuration information can also contain data with an interval of validity smaller than one full run, for example the LVL1 prescales may vary on a luminosity block (order of 60 seconds) base. This would simply result in several LVL1 prescale objects in the file header, one for each luminosity block.

Following the ATLAS event data model policy, the eventwise trigger decision class is transient/persistent separated. This means that the trigger decision class written to disk (persistent) and the one in the transient memory are not the same. Main benefits are: Firstly, schema evolution (allows reading of any old data from disk). Secondly, it saves disk space (compression of data).

\section{USER INTERFACE TO THE TRIGGeR}

Providing one common place to query for any trigger related information, the trigger-user interface (TUI) was developed this year and is now routinely used in many physics and trigger studies. It allows for accessing all event-wise trigger decision information but also the run-wise configuration data. Combining the trigger configuration with the raw event-wise data, the TUI provides means to query for human-readable trigger names instead of chain counters or bit positions. Fig. 2 depicts the design of the TUI which is implemented as a standard ATLAS software tool. For every event, the TUI tool requests the raw event-wise trigger decision. Internally, the persistent trigger data class is read and converted into the requested transient class. Configuration data is provided by a service which is shared with the trigger-online software. Several implementations of this service, all sharing the same interface, exist hence allowing to run in various scenarios, as will be explained below. Merging and mapping of the configuration with the raw trigger decision data happens only on demand (when needed because of user queries to the TUI tool). Once the TUI tool has built such a mapping, it is cached into the transient trigger data class to speed-up further queries.

In the following list the main TUI use-cases are summarised.

- Trigger aware analysis: the TUI is used to check whether events passed a desired trigger (query by names or counter). Further, the TUI tool can be used to query prescale and pass-through factors and flags; study trigger efficiencies, and do luminosity calculations

- Trigger studies: Retrieve trigger objects (e.g. electrons, tracks, clusters) that caused the accept or that failed. Link together LVL1, LVL2, and EF triggers, e.g. follow the refinement of a trigger electron through the trigger levels and steps; compare trigger objects with offline objects.

In the use-cases above, the configuration can be taken directly from the AOD header. In certain situations, however, it is important to read the configuration from the TriggerDB or local XML files. This is realised by changing the default configuration service. This allows to cross check and debug the trigger decision, the trigger configuration data flow, and all software involved against the original online trigger configuration (TriggerDB). Furthermore, it makes trigger optimisation studies more convenient:
- Trigger optimisation: Vary some trigger selection cuts and re-run the HLT. This requires a modified trigger menu (configuration). Then, compare the new trigger result to the previous one.

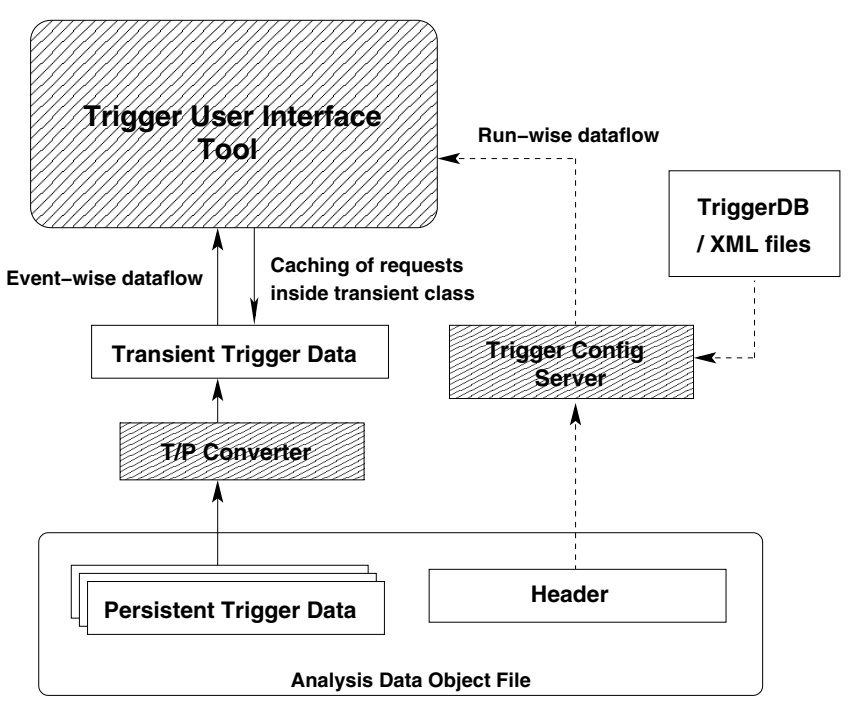

Fig. 2. The user interface of the ATLAS trigger. Event-wise trigger data is retrieved after T/P conversion. The trigger configuration is read through a service to allow transparent reading from different sources.

\section{TRigger Results in Physics Studies}

Easy access to trigger data is essential in many situations, ranging from physics analyses to online event displays. A brief description of three clients of the trigger-user interface is given in the following list.

\section{A. Trigger Aware Analyses}

All physics analyses are based on one or several triggers. As ATLAS is nearing completion more and more of the Monte Carlo analyses are starting to look into the trigger decision. In a close liaison between the ATLAS physics groups and the trigger groups, relevant triggers are built and optimised in terms of thresholds and available bandwidth.

\section{B. Trigger Rate Estimation}

In the process of building a draft trigger menu for initial data taking, the calculation of trigger rates is the main handle for setting thresholds, prescales etc. The official ATLAS tool for calculating the trigger rates is based on the trigger-user interface. For statistics reasons, all prescales are turned off and only incorporated again in the very end. This way, more events survive the trigger selection thus allowing to run on less Monte Carlo data.

\section{Event Display}

Two of the three general ATLAS event displays (Atlantis and VP1) already exploit trigger data provided by the triggeruser interface tool. One can display the passed triggers for all levels and see the RoI position in the detector geometry. It is foreseen to also detail trigger objects as electrons, muons, clusters and so on. 


\section{CONCLUSIONS}

This paper described the ATLAS trigger operation and in particular the trigger-user interface, focusing on the accessibility it provides to both online quantities and the trigger configuration.

As ATLAS is nearing completion, more and more Monte Carlo physics studies start to incorporate the trigger system. At the same time the ATLAS Trigger and Data Acquisition group is studying the performance, efficiencies, rates etc. of all triggers aiming at building a complete trigger menu for initial data taking. In all these places, the trigger-user interface tool is successfully used.

\section{ACKNOWLEDGMENT}

It is a pleasure to thank the help and friendly collaboration of our colleagues in the ATLAS Trigger and Data Acquisition group.

\section{REFERENCES}

[1] Jenni, P et al 1999 ATLAS detector and physics performance Technical Design Report, part 1 ser. Technical Design Report ATLAS (Geneva: CERN)

[2] Jenni, P et al 1999 ATLAS detector and physics performance Technical Design Report, part 2 ser. Technical Design Report ATLAS (Geneva: CERN)

[3] Jenni, P et al 2003 ATLAS high-level trigger, data-acquisition and controls Technical Design Report ser. Technical Design Report ATLAS (Geneva: CERN)

[4] Stelzer, J et al 2007 The configuration system of the ATLAS Trigger Presented at International Conference on Computing in High Energy and Nuclear Physics 2007 (CHEP 07) 